\title{
Exact solutions involving special functions for unsteady convective flow of magnetohydrodynamic second grade fluid with ramped conditions
}

\author{
Muhammad Bilal Riaz',2, Kashif Ali Abro ${ }^{2,3}$, Khadijah M. Abualnaja4 , Ali Akgül5 , Aziz Ur Rehman', \\ Muhammad Abbas ${ }^{6 *}$ (D) and Y.S. Hamed ${ }^{4}$
}

\author{
${ }^{*}$ Correspondence: \\ muhammad.abbas@uos.edu.pk \\ ${ }^{6}$ Department of Mathematics, \\ University of Sargodha, 40100 \\ Sargodha, Pakistan \\ Full list of author information is \\ available at the end of the article
}

\begin{abstract}
A number of mathematical methods have been developed to determine the complex rheological behavior of fluid's models. Such mathematical models are investigated using statistical, empirical, analytical, and iterative (numerical) methods. Due to this fact, this manuscript proposes an analytical analysis and comparison between Sumudu and Laplace transforms for the prediction of unsteady convective flow of magnetized second grade fluid. The mathematical model, say, unsteady convective flow of magnetized second grade fluid, is based on nonfractional approach consisting of ramped conditions. In order to investigate the heat transfer and velocity field profile, we invoked Sumudu and Laplace transforms for finding the hidden aspects of unsteady convective flow of magnetized second grade fluid. For the sake of the comparative analysis, the graphical illustration is depicted that reflects effective results for the first time in the open literature. In short, the obtained profiles of temperature and velocity fields with Laplace and Sumudu transforms are in good agreement on the basis of numerical simulations.
\end{abstract}

Keywords: Integral transform; MHD; Unsteady convective flow; Special functions

\section{Introduction}

The natural convection heat transfer from a vertical plate to a fluid has implementations in many industrial processes. The investigators have applied different sets of thermal conditions at the bounding plate. Ganesan et al. [1] have described the solutions for velocity and temperature applying continuous and well-defined conditions at the wall. Samiulhaq et al. [2] have presented the influence of radiation and porosity on the unsteady magnetohydrodynamic (MHD) flow. Chandran et al. [3] have worked on the unsteady free convection flow of an incompressible viscous fluid near a vertical plate with ramped wall temperature. Seth et al. $[4,5]$ have obtained the exact solutions of the MHD natural convection flow.

(c) The Author(s) 2021. This article is licensed under a Creative Commons Attribution 4.0 International License, which permits use, sharing, adaptation, distribution and reproduction in any medium or format, as long as you give appropriate credit to the original author(s) and the source, provide a link to the Creative Commons licence, and indicate if changes were made. The images or other third party material in this article are included in the article's Creative Commons licence, unless indicated otherwise in a credit line to the material. If material is not included in the article's Creative Commons licence and your intended use is not permitted by statutory regulation or exceeds the permitted use, you will need to obtain permission directly from the copyright holder. To view a copy of this licence, visit http://creativecommons.org/licenses/by/4.0/. 
Over the set of functions [6],

$$
A=\left\{r(t)\left|\exists N, \tau_{1}, \tau_{2}>0,\right| r(t) \mid<N \exp \left(|t| / \tau_{j}\right), \text { if } t \in(-1)^{j} \times[0, \infty)\right\},
$$

the Sumudu transform is defined as

$$
R(u)=S[r(t)]=\int_{0}^{\infty} r(u t) \exp (-t) d t, \quad u \in\left(-\tau_{1}, \tau_{2}\right) .
$$

The Sumudu transform method (STM) was started with Watugala [7] when he researched the engineering control problems. The implementations of the Sumudu transform method of the partial differential equations have been discussed in the literature [8]. Weerakoon [9] has investigated a complex inversion formula for the Sumudu transform. This transformation was initially discussed to be a theoretical dual of the Laplace transform. The Sumudu transform has very valuable features in the implementations of sciences and engineering. This transform has been utilized to investigate many problems without resorting to a new frequency domain having scale and unit-preserving features. Integro-differential equations have been investigated by Sumudu transform in [10]. Watugala [11] has investigated the transform for two variables with the emphasis on solutions to partial differential equations. Belgacem et al. [12,13] have discussed the convolution-type integral equations with the focus on production problems. For more details, see [14-18].

We construct our paper as follows: We present the mathematical modeling of the problem in Sect. 2. We discuss the solution of the problem in Sect. 3. We give an alternative method in Sect. 4. We present the discussion in Sect. 5. We give the conclusion in the last section.

\section{Mathematical modeling}

Let us assume that the unsteady MHD, natural convection, time dependent, incompressible viscous flow of second grade fluid near an infinite vertical plate is embedded in a porous medium with ramped wall temperature. In this case, we consider the Cartesian coordinate system. The plate is placed in the $(x, y)$ plane with $x$-axis oriented vertically and the $y$-axis in the normal direction. At the end of the wall, velocity and temperature are time dependent with certain limits of time identified as the characteristic time; velocity and temperature after that time attain constant values $V_{0}$ and $T_{\infty}$, respectively. The fundamental governing partial differential equations with small Reynolds number and usual Boussinesq's approximation are given as [19-22]:

$$
\begin{aligned}
\frac{\partial V(y, t)}{\partial t}= & v\left(1+\frac{\alpha_{1}}{\mu} \frac{\partial}{\partial t}\right) \frac{\partial^{2} V(y, t)}{\partial y^{2}}+g \beta\left(T(y, t)-T_{\infty}\right) \\
& -\left[\frac{\sigma_{0} M_{0}^{2}}{\rho}+\frac{v \phi}{k_{0}}\left(1+\frac{\alpha_{1}}{\mu} \frac{\partial}{\partial t}\right)\right] V(y, t), \\
\frac{\partial T(y, t)}{\partial t}= & \frac{k}{\rho C_{p}}\left(1+\frac{16 \sigma_{1} T_{\infty}^{3}}{3 k K_{1}}\right) \frac{\partial^{2} T(y, t)}{\partial y^{2}},
\end{aligned}
$$

where $V(y, t), T(y, t), \rho, v, \alpha_{1}, \beta_{T}, g, k$, and $C_{p}$ denote the fluid velocity, temperature of the fluid, density, kinematic viscosity, second grade parameter, coefficient of volumetric 
thermal expansion, gravitational acceleration, thermal conductivity, and heat capacity at constant pressure, respectively.

The appropriate initial and boundary conditions are presented as:

$$
\begin{aligned}
& V(y, 0)=0, \quad T(y, 0)=T_{\infty}, \quad \frac{\partial V(y, 0)}{\partial t}=0, \quad y \geq 0, \\
& V(0, t)=f_{1}(t), \quad T(0, t)=f_{2}(t),
\end{aligned}
$$

where

$$
\begin{aligned}
& f_{1}(t)=\left\{\begin{array}{ll}
V_{0} \frac{t}{t_{0}}, & 0<t \leq t_{0}, \\
V_{0}, & t>t_{0},
\end{array} \text { and } f_{2}(t)= \begin{cases}T_{\infty}+\left(T_{w}-T_{\infty}\right) \frac{t}{t_{0}}, & 0<t \leq t_{0}, \\
T(0, t)=T_{w}, & t>t_{0},\end{cases} \right. \\
& V(y, t) \rightarrow 0, \\
& \quad T(y, t) \rightarrow \infty, \quad \text { as } y \rightarrow \infty .
\end{aligned}
$$

Introducing the following dimensionless variables:

$$
\begin{aligned}
& \psi=\frac{V_{0}}{v} y, \quad t^{*}=\frac{V_{0}^{2}}{v} t, \quad V^{*}=\frac{V}{V_{0}}, \quad \theta=\frac{T-T_{\infty}}{T_{w}-T_{\infty}}, \quad G_{r}=\frac{g \beta t_{0}\left(T-T_{\infty}\right)}{V_{0}^{3}}, \\
& N r=\frac{16 \sigma_{1} T_{\infty}^{3}}{3 k K_{1}}, \quad M=\frac{\sigma_{0} M_{0}^{2} v}{\rho V_{0}^{2}}, \quad \operatorname{Pr}=\frac{v C_{p}}{k}, \quad P r_{0}=\frac{P r}{1+N r}, \\
& \alpha=\frac{\alpha_{1} \rho V_{0}^{2}}{\mu^{2}}, \quad \frac{1}{K}=\frac{v^{2} \phi}{k_{0} V_{0}^{2}}, \quad a=M+\frac{1}{K}, \quad b=\frac{\alpha}{K},
\end{aligned}
$$

and removing the star notation, the required dimensionless momentum and energy equations are obtained as:

$$
\begin{aligned}
& \frac{\partial V(\psi, t)}{\partial t}=\frac{\partial^{2} V(\psi, t)}{\partial \psi^{2}}+G_{r} \theta(\psi, t)-a V(\psi, t)-b \frac{\partial V(\psi, t)}{\partial t}+\alpha \frac{\partial^{3} V(\psi, t)}{\partial t \partial \psi^{2}}, \\
& \frac{\partial \theta(\psi, t)}{\partial t}=\frac{1}{P r_{0}} \frac{\partial^{2} \theta(\psi, t)}{\partial \psi^{2}}
\end{aligned}
$$

and the corresponding initial and boundary conditions are presented as

$$
\begin{aligned}
& V(\psi, 0)=0, \quad \theta(\psi, 0)=0,\left.\quad \frac{\partial V(\psi, t)}{\partial t}\right|_{t=0}=0, \\
& V(0, t)=f(t), \quad \theta(0, t)=f(t), \quad \text { where } f(t)= \begin{cases}t, & 0<t \leq 1, \\
1, & t>1,\end{cases} \\
& V(\psi, t) \rightarrow 0, \quad \theta(\psi, t) \rightarrow 0 \quad \text { as } \psi \rightarrow \infty .
\end{aligned}
$$

Also, a new version of Sumudu transform definition in modified form due to Watugala [7] is presented as

$$
R(u)=\mathbb{S}[r(t)]=\int_{0}^{\infty} \frac{r(t) e^{-\frac{t}{u}}}{u} d t
$$


Theorem 1 ([14]) If $R(u)$ is the Sumudu transform of $r(t)$, then the Sumudu transform of the derivatives with integer order is as follows:

$$
\mathbb{S}\left[\frac{d^{n} r(t)}{d t^{n}}\right]=u^{-n}\left[R(u)-\left.\sum_{\eta=0}^{n-1} u^{\eta} \frac{d^{n} r(t)}{d t^{n}}\right|_{t=0}\right] .
$$

Proof The Sumudu transform of the first derivative of $r(t), r^{\prime}(t)=d r(t) / d t$, is given by

$$
\begin{aligned}
\mathbb{S}\left[\frac{d r(t)}{d t}\right] & =\int_{0}^{\infty} e^{-t} \frac{d r(u t)}{d t} d t=\lim _{\xi \rightarrow \infty} \int_{0}^{\xi} e^{-t} \frac{d r(u t)}{d t} d t \\
& =\lim _{\xi \rightarrow \infty}\left[\left.\frac{1}{u} e^{-\frac{t}{u}} r(t)\right|_{0} ^{\xi}+\frac{1}{u^{2}} \int_{0}^{\xi} e^{-\frac{t}{u}} r(t) d t\right] \\
& =\lim _{\xi \rightarrow \infty}\left[\left.\frac{1}{u} e^{-\frac{t}{u}} r(t)\right|_{0} ^{\xi}+\frac{1}{u}\left(\frac{1}{u} \int_{0}^{\xi} e^{-\frac{t}{u}} r(t) d t\right)\right] \\
& =\lim _{\xi \rightarrow \infty}\left[-\frac{1}{u} r(0)+\frac{1}{u}\left(\frac{1}{u} \int_{0}^{\xi} e^{-\frac{t}{u}} r(t) d t\right)\right] \\
& =-\frac{1}{u} r(0)+\frac{1}{u} R(u) .
\end{aligned}
$$

To get the Sumudu transformation for the second order derivative of the function $r(t)$, proceeding in the same way, we obtain

$$
\mathbb{S}\left[\frac{d^{2} r(t)}{d t^{2}}\right]=\frac{1}{u^{2}}\left[R(u)-r(0)-\left.u \frac{d r(t)}{d t}\right|_{t=0}\right] .
$$

To derive the general formula from this theorem for Sumudu transform of any integer order $n$, using mathematical induction, we get

$$
\mathbb{S}\left[\frac{d^{n} r(t)}{d t^{n}}\right]=u^{-n}\left[R(u)-\left.\sum_{\eta=0}^{n-1} u^{\eta} \frac{d^{n} r(t)}{d t^{n}}\right|_{t=0}\right]
$$

which completes the proof.

Next, defined for $\operatorname{Re}(s)>0$, the Laplace transform for the function $r(t)$ is given by

$$
F(s)=\mathcal{L}[r(t)]=\int_{0}^{\infty} e^{-s t} r(t) d t
$$

In consideration of the definition in Eq. (14), the Laplace and Sumudu transforms exhibit a duality relation which is expressed in the following way:

$$
R\left(\frac{1}{s}\right)=s F(s), \quad F\left(\frac{1}{u}\right)=u R(u)
$$

which referred as Sumudu-Laplace duality and illustrates the fact that Laplace and Sumudu transformations interchange the images of Heaviside function $H(t)$ and Dirac function $\delta(t)$, since

$$
\mathcal{L}[\delta(t)]=\mathbb{S}[H(t)]=1, \quad \mathcal{L}[H(t)]=\mathbb{S}[\delta(t)]=\frac{1}{u} .
$$


Similarly, for the functions $\cos (t)$ and $\sin (t)$, we have

$$
\mathcal{L}[\sin (t)]=\mathbb{S}[\cos (t)]=\frac{1}{1+u^{2}}, \quad \mathcal{L}[\cos (t)]=\mathbb{S}[\sin (t)]=\frac{u}{1+u^{2}},
$$

which is also consistent for the established result in Theorem 1 and integration formulas:

$$
\begin{aligned}
& \mathbb{S}\left[r^{\prime}(t)\right]=\frac{\mathbb{S}[r(t)]-r(0)}{u}, \\
& \mathbb{S}\left[\int_{0}^{t} r(\tau) d \tau\right]=u \mathbb{S}[r(t)] .
\end{aligned}
$$

The next theorem is very helpful for finding the solution of differential equations involving multiple integrals by using Sumudu transformation efficiently.

Theorem 2 ([13]) Let $r(t)$ be in A. The Sumudu transform $R^{n}(u)$ of the nth antiderivative of $r(t)$, obtained by $n$ times successively integrating the function $r(t)$,

$$
G^{n}(t)=\iint_{0}^{t} \cdots \int_{0}^{t} r(\tau)(d \tau)^{n}
$$

can be obtained, for $n \geq 1$, as

$$
R^{n}(u)=\mathbb{S}\left(G^{n}(t)\right)=u^{n} R(u) .
$$

Proof For $n=1$, Eq. (26) holds due to Eq. (24). To prove this theorem by induction, suppose that Eq. (26) holds for some $n$, and we prove it also holds for $n+1$. Again using Eq. (24), we have

$$
R^{n+1}(u)=\mathbb{S}\left(G^{n+1}(t)\right)=\mathbb{S}\left[\int_{0}^{t} G^{n}(\tau) d \tau\right]=u \mathbb{S}\left(G^{n}(t)\right)=u\left[u^{n} R(u)\right]=u^{n+1} R(u) .
$$

This theorem generalizes the Sumudu convolution Theorem 4.1 as presented in Belgacem et al. [12], which states that the convolution of two functions $g$ and $h$, defined as

$$
(g \star h)(t)=\int_{0}^{t} g(\tau) h(t-\tau) d \tau
$$

has its Sumudu transformation given by

$$
\mathbb{S}((g \star h)(t))=u G(u) H(u) .
$$

Similarly, the Sumudu transform of $\left(h_{1} \star h_{2} \star h_{3}\right)$, with $h_{1}, h_{2}, h_{3}$ in $A$, is given by

$$
\mathbb{S}\left(\left(h_{1} \star h_{2} \star h_{3}\right)(t)\right)=u^{2} H_{1}(u) H_{2}(u) H_{3}(u)
$$

\section{Solution of the problem}

In this section, the Sumudu transformation method is used to get the solution of the considered problem. 


\subsection{Exact solution of heat profile by Sumudu transformation}

Theorem 3 Let $\mathbb{S}$ be the Sumudu operator. Applying this operator on equation (10), along with initial and boundary conditions (11), (12) and (13), the exact solution of heat profile is

$$
\theta(\psi, t)=\theta_{1}(\psi, t)-\theta_{2}(\psi, t)
$$

where

$$
\begin{aligned}
& \theta_{1}(\psi, t)=\mathbb{S}^{-1}\left(u e^{-\frac{a_{0}}{\sqrt{u}}}\right)=\int_{0}^{\tau} f(t) d(t) \text { with } f(t)=\operatorname{erfc}\left(\frac{a_{0}}{2 \sqrt{t}}\right), a_{0}=\sqrt{P_{r_{0}}} \psi, \\
& \theta_{2}(\psi, t)=\theta_{1}(\psi, \tau-1) H(\tau-1) .
\end{aligned}
$$

Proof Applying the Sumudu transformation technique to get the solution of Eq. (10) and taking into consideration Eq. (18) with given boundary conditions yields

$$
\frac{d^{2} \bar{\theta}(\psi, u)}{d \psi^{2}}-\frac{P_{r_{0}}}{u} \bar{\theta}(\psi, u)=0,
$$

with

$$
\bar{\theta}(\psi, u) \rightarrow 0, \quad \text { as } \psi \rightarrow \infty \quad \text { and } \quad \bar{\theta}(0, u)=u\left(1-e^{-\frac{1}{u}}\right)
$$

and its solution is given by

$$
\bar{\theta}(\psi, u)=u\left(1-e^{-\frac{1}{u}}\right) e^{-\psi \sqrt{\frac{P r_{0}}{u}}} .
$$

Further, it can be written as

$$
\bar{\theta}(\psi, u)=\left(u e^{-\frac{a_{0}}{\sqrt{u}}}\right)-e^{-\frac{1}{u}}\left(u e^{-\frac{a_{0}}{\sqrt{u}}}\right), \quad \text { where } a_{0}=\sqrt{P_{r_{0}}} \psi .
$$

Applying the Sumudu inverse transformation gives the solution

$$
\theta(\psi, t)=\theta_{1}(\psi, t)-\theta_{2}(\psi, t)
$$

where

$$
\begin{aligned}
& \theta_{1}(\psi, t)=\mathbb{S}^{-1}\left(u e^{-\frac{a_{0}}{\sqrt{u}}}\right)=\int_{0}^{\tau} f(t) d(t) \text { and } f(t)=\operatorname{erfc}\left(\frac{a_{0}}{2 \sqrt{t}}\right), \\
& \theta_{2}(\psi, t)=\theta_{1}(\psi, \tau-1) H(\tau-1) .
\end{aligned}
$$

\subsection{Exact solution of heat profile by Laplace transformation}

Theorem 4 Let $\mathscr{L}$ be the Laplace operator. Applying this operator on equation (10), along with initial and boundary conditions (11), (12), and (13), the exact solution of heat profile is

$$
\theta(\psi, t)=\theta_{r}(\psi, t)-\theta_{r}\left(\psi, \tau_{0}\right) H\left(\tau_{0}\right),
$$


where

$$
\theta_{r}(\psi, t)=\left(\frac{P r_{0}}{2} \psi^{2}+t\right) \operatorname{erfc}\left(\sqrt{\frac{P r_{0}}{4 t}} \psi\right)-\left(\sqrt{\frac{P r_{0} t}{\pi}} \psi\right) e^{\frac{-P r_{0} \psi^{2}}{4 t}}
$$

and $H\left(\tau_{0}\right)$ represents a standard Heaviside function with $\tau_{0}=t-1$.

Proof Applying Laplace transformation to get the solution of Eq. (10) and using appropriate boundary conditions yields

$$
\frac{\partial^{2} \bar{\theta}(\psi, m)}{\partial \psi^{2}}-m P r_{0} \bar{\theta}(\psi, m)=0
$$

The solution of above differential equation (37) is obtained as

$$
\bar{\theta}(\psi, m)=c_{1} e^{\psi \sqrt{m P r_{0}}}+c_{2} e^{-\psi \sqrt{m P r_{0}}} .
$$

Applying the conditions to find unknowns $c_{1}$ and $c_{2}$ yields

$$
\bar{\theta}(\psi, m) \rightarrow 0 \quad \text { as } \psi \rightarrow \infty \quad \text { and } \quad \bar{\theta}(0, m)=\left(\frac{1-e^{-m}}{m^{2}}\right) .
$$

We get

$$
\begin{aligned}
& \bar{\theta}(\psi, m)=\left(\frac{1-e^{-m}}{m^{2}}\right) e^{-\psi \sqrt{m P r_{0}}} \\
& \bar{\theta}(\psi, m)=\left(\frac{e^{-\psi \sqrt{m P r_{0}}}}{m^{2}}\right)-e^{-m}\left(\frac{e^{-\psi \sqrt{m P r_{0}}}}{m^{2}}\right)=\bar{\theta}_{r}(\psi, m)-e^{-m} \bar{\theta}_{r}(\psi, m) .
\end{aligned}
$$

After applying inverse Laplace transformation on Eq. (40), we get

$$
\begin{aligned}
& \theta(\psi, t)=\theta_{r}(\psi, t)-\theta_{r}\left(\psi, \tau_{0}\right) H\left(\tau_{0}\right), \\
& \theta_{r}(\psi, t)=\left(\frac{P r_{0}}{2} \psi^{2}+t\right) \operatorname{erfc}\left(\sqrt{\frac{P r_{0}}{4 t}} \psi\right)-\left(\sqrt{\frac{P r_{0} t}{\pi}} \psi\right) e^{\frac{-P r_{0} \psi^{2}}{4 t}},
\end{aligned}
$$

where $H\left(\tau_{0}\right)$ represents a standard Heaviside function with $\tau_{0}=t-1$.

\subsection{Solution of velocity profile}

Theorem 5 Let $\mathbb{S}$ be the Sumudu operator. Applying this operator on equation (9), along with initial and boundary conditions (11), (12), and (13), the exact solution of velocity profile is given in equation (62).

Proof The solution of Eq. (9) by using Sumudu transformation is obtained from

$$
\left(1+\frac{\alpha}{u}\right) \frac{d^{2} \bar{V}(\psi, u)}{d \psi^{2}}-\left(\frac{1}{u}+a+\frac{b}{u}\right) \bar{V}(\psi, u)=-G_{r} \bar{\theta}(\psi, u) .
$$

Its general solution can be written as

$$
\bar{V}(\psi, u)=c_{1} e^{\psi \sqrt{\frac{a u+1+b}{u+\alpha}}}+c_{2} e^{-\psi \sqrt{\frac{a u+1+b}{u+\alpha}}}-\left(\frac{G_{r} u^{3}\left(1-e^{-\frac{1}{u}}\right) e^{-\psi \sqrt{\frac{P_{r 0}}{u}}}}{(u+\alpha) P_{r_{0}}-u(a u+1+b)}\right) .
$$


Since $\bar{V}(\psi, u) \rightarrow 0$ as $\psi \rightarrow \infty$ and $\bar{V}(0, u)=u\left(1-e^{-\frac{1}{u}}\right)$, we get

$$
\begin{aligned}
\bar{V}(\psi, u)= & u\left(1-e^{-\frac{1}{u}}\right) e^{-\psi \sqrt{\frac{a u+1+b}{u+\alpha}}} \\
& +\frac{G_{r} u^{3}\left(1-e^{-\frac{1}{u}}\right)}{(u+\alpha) P_{r_{0}}-u(a u+1+b)}\left[e^{-\psi \sqrt{\frac{a u+1+b}{u+\alpha}}}-e^{-\psi \sqrt{\frac{P_{r_{0}}}{u}}}\right] \\
\bar{V}(\psi, u)= & \bar{v}_{1}(\psi, u)+\bar{v}_{2}(\psi, u) .
\end{aligned}
$$

Applying Sumudu inverse transformation gives

$$
V(\psi, t)=\mathbb{S}^{-1}(\bar{V}(\psi, u))=\mathbb{S}^{-1}\left(\bar{v}_{1}(\psi, u)\right)+\mathbb{S}^{-1}\left(\bar{v}_{2}(\psi, u)\right),
$$

where

$$
\begin{aligned}
& \bar{v}_{1}(\psi, u)=u\left(1-e^{-\frac{1}{u}}\right) e^{-\psi \sqrt{\frac{a u+c}{u+\alpha}}}, \quad c=1+b, \\
& \bar{v}_{2}(\psi, u)=\frac{G_{r} u^{3}\left(1-e^{-\frac{1}{u}}\right)}{(u+\alpha) P_{r_{0}}-u(a u+c)}\left[e^{-\psi \sqrt{\frac{a u+c}{u+\alpha}}}-e^{-\psi \sqrt{\frac{P_{r_{0}}}{u}}}\right], \\
& \bar{v}_{1}(\psi, u)=\bar{v}_{11}(\psi, u)-\bar{v}_{12}(\psi, u), \\
& \mathbb{S}^{-1}\left(\bar{v}_{1}(\psi, u)\right)=v_{11}(\psi, t)-v_{12}(\psi, t),
\end{aligned}
$$

where $*$ denotes the convolution. Then, we have

$$
\begin{aligned}
& \nu_{11}(\psi, t)=\mathbb{S}^{-1}\left(u e^{-\psi \sqrt{\frac{a u+c}{u+\alpha}}}\right)=\int_{0}^{t} f_{2}(\tau) d \tau, \\
& v_{12}(\psi, t)=\mathbb{S}^{-1}\left[e^{\frac{-1}{u}}\left(u e^{-\psi \sqrt{\frac{a u+c}{u+\alpha}}}\right)\right] \\
& =v_{11}(\psi, t-1) H(t-1), \\
& F_{2}(u)=e^{-\psi \sqrt{\frac{a u+c}{u+\alpha}}} .
\end{aligned}
$$

It is complicated to find the Sumudu inverse of $F_{2}(u)$ in exponential form, so we have to express it in its equivalent form as

$$
\begin{aligned}
& F_{2}(u)=\sum_{n_{1}=0}^{\infty} \sum_{n_{2}=0}^{\infty} \sum_{n_{3}=0}^{\infty} \frac{(-1)^{n_{3}} a^{\frac{n_{1}}{2}-n_{2}}(-\psi)^{n_{1}} d^{n_{2}} \Gamma\left(\frac{n_{1}}{2}+1\right) \Gamma\left(n_{2}+n_{3}\right)}{n_{1} ! n_{2} ! n_{3} !(\alpha)^{n_{2}+n_{3}} \Gamma\left(\frac{n_{1}}{2}+1-n_{2}\right) \Gamma\left(n_{2}\right)} u^{n_{3}}, \quad \text { and } \\
& d=c-a \alpha .
\end{aligned}
$$

Applying Sumudu inverse transformation gives

$$
\begin{aligned}
& f_{2}(t)=\mathbb{S}^{-1}\left(F_{2}(u)\right)=\sum_{n_{1}=0}^{\infty} \sum_{n_{2}=0}^{\infty} \sum_{n_{3}=0}^{\infty} \frac{(-1)^{n_{3}} a^{\frac{n_{1}}{2}-n_{2}}(-\psi)^{n_{1}} d^{n_{2}} \Gamma\left(\frac{n_{1}}{2}+1\right) \Gamma\left(n_{2}+n_{3}\right)}{n_{1} ! n_{2} !\left(n_{3} !\right)^{2}(\alpha)^{n_{2}+n_{3}} \Gamma\left(\frac{n_{1}}{2}+1-n_{2}\right) \Gamma\left(n_{2}\right)} t^{n_{3}}, \\
& \bar{v}_{2}(\psi, u)=\frac{G r}{a} u\left[-1+\frac{D}{1-a_{2} u}+\frac{E}{1+b_{2} u}\right]\left(1-e^{-\frac{1}{u}}\right)\left[e^{-\psi \sqrt{\frac{a u+c}{u+\alpha}}}-e^{-\psi \sqrt{\frac{P_{0}}{u}}}\right], \\
& \bar{v}_{2}(\psi, u)=\frac{G r}{a} u\left[-1+D F_{3}(u)+E F_{4}(u)\right]\left(1-e^{-\frac{1}{u}}\right)\left[F_{2}(u)-F_{1}(u)\right]
\end{aligned}
$$




$$
\begin{aligned}
\bar{v}_{2}(\psi, u)= & \frac{G r}{a}\left[-u F_{2}(u)+u F_{1}(u)+e^{-\frac{1}{u}}\left(u F_{2}(u)\right)-e^{-\frac{1}{u}}\left(u F_{1}(u)\right)\right] \\
& +\frac{G r D}{a}\left[u F_{2}(u) F_{3}(u)-u F_{1}(u) F_{3}(u)\right. \\
& \left.-e^{-\frac{1}{u}}\left(u F_{2}(u) F_{3}(u)\right)+e^{-\frac{1}{u}}\left(u F_{1}(u) F_{3}(u)\right)\right] \\
& +\frac{G r E}{a}\left[u F_{2}(u) F_{4}(u)-u F_{1}(u) F_{4}(u)\right. \\
& \left.-e^{-\frac{1}{u}}\left(u F_{2}(u) F_{4}(u)\right)+e^{-\frac{1}{u}}\left(u F_{1}(u) F_{4}(u)\right)\right],
\end{aligned}
$$

with

$$
\begin{aligned}
& j=\frac{P_{r_{0}}-c}{a}, \quad r=\frac{\alpha P_{r_{0}}}{a}, \quad g=\frac{j}{2}, \quad h=\sqrt{r+g^{2}}, \quad a_{2}=\frac{1}{h+g}, \\
& b_{2}=\frac{1}{h-g}, \quad D=\frac{a_{2} r+j}{\left(a_{2}+b_{2}\right)\left(h^{2}-g^{2}\right)}, \quad E=\frac{b_{2} r-j}{\left(a_{2}+b_{2}\right)\left(h^{2}-g^{2}\right)}, \\
& F_{1}(u)=e^{-\psi \sqrt{\frac{P_{r_{0}}}{u}}}, \quad F_{2}(u)=e^{-\psi \sqrt{\frac{a u+c}{u+\alpha}}}, \\
& F_{3}(u)=\frac{1}{1-a_{2} u}, \quad F_{4}(u)=\frac{1}{1+b_{2} u} .
\end{aligned}
$$

After applying the Sumudu inverse transformation, we obtain

$$
\begin{aligned}
v_{2}(\psi, t)= & \frac{G r}{a}\left[-\Phi_{1}(\psi, t)+\Phi_{2}(\psi, t)+\Phi_{1}(\psi, t-1) H(t-1)-\Phi_{2}(\psi, t-1) H(t-1)\right] \\
& +\frac{G r D}{a}\left[\Phi_{3}(\psi, t)-\Phi_{4}(\psi, t)-\Phi_{3}(\psi, t-1) H(t-1)\right. \\
& \left.+\Phi_{4}(\psi, t-1) H(t-1)\right] \\
& +\frac{G r E}{a}\left[\Phi_{5}(\psi, t)-\Phi_{6}(\psi, t)-\Phi_{5}(\psi, t-1) H(t-1)\right. \\
& \left.+\Phi_{6}(\psi, t-1) H(t-1)\right],
\end{aligned}
$$

where

$$
\begin{aligned}
& \Phi_{1}(\psi, t)=\int_{0}^{t} f_{2}(\tau) d(\tau), \\
& \Phi_{2}(\psi, t)=\int_{0}^{t} f_{1}(\tau) d(\tau), \\
& \Phi_{3}(\psi, t)=\left(f_{2} * f_{3}\right)(t)=\int_{0}^{t} f_{2}(\tau) f_{3}(t-\tau) d(\tau), \\
& \Phi_{4}(\psi, t)=\left(f_{1} * f_{3}\right)(t)=\int_{0}^{t} f_{1}(\tau) f_{3}(t-\tau) d(\tau), \\
& \Phi_{5}(\psi, t)=\left(f_{2} * f_{4}\right)(t)=\int_{0}^{t} f_{2}(\tau) f_{4}(t-\tau) d(\tau), \\
& \Phi_{6}(\psi, t)=\left(f_{1} * f_{4}\right)(t)=\int_{0}^{t} f_{1}(\tau) f_{4}(t-\tau) d(\tau), \\
& f_{1}(t)=\operatorname{erfc}\left(\frac{a_{1}}{2 \sqrt{t}}\right), \quad \text { with } a_{1}=\sqrt{P_{r_{0}}} \psi,
\end{aligned}
$$




$$
\begin{aligned}
& f_{2}(t)=\sum_{n_{1}=0}^{\infty} \sum_{n_{2}=0}^{\infty} \sum_{n_{3}=0}^{\infty} \frac{(-1)^{n_{3}} a^{\frac{n_{1}}{2}-n_{2}}(-\psi)^{n_{1}} d^{n_{2}} \Gamma\left(\frac{n_{1}}{2}+1\right) \Gamma\left(n_{2}+n_{3}\right)}{n_{1} ! n_{2} !\left(n_{3} !\right)^{2}(\alpha)^{n_{2}+n_{3}} \Gamma\left(\frac{n_{1}}{2}+1-n_{2}\right) \Gamma\left(n_{2}\right)} t^{n_{3}}, \\
& f_{3}(t)=e^{a_{2} t} \\
& f_{4}(t)=e^{-b_{2} t} .
\end{aligned}
$$

\section{Alternative method to calculate $\bar{v}_{2}(\psi, u)$ by using discrete convolution (the}

\section{Cauchy product)}

$$
\begin{aligned}
\bar{v}_{2}(\psi, u)= & \frac{G_{r} u^{3}\left(1-e^{-\frac{1}{u}}\right)}{(u+\alpha) P_{r_{0}}-u(a u+c)}\left[e^{-\psi \sqrt{\frac{a u+c}{u+\alpha}}}-e^{-\psi \sqrt{\frac{P r_{0}}{u}}}\right], \\
\bar{v}_{2}(\psi, u)= & \frac{G r}{a}\left[-u-j+\frac{1}{h^{2}-g^{2}}\left\{\frac{D}{1-a_{2} u}+\frac{E}{1+b_{2} u}\right\}\right] \\
& \times\left(1-e^{-\frac{1}{u}}\right)\left[e^{-\psi \sqrt{\frac{a u+c}{u+\alpha}}}-e^{-\psi \sqrt{\frac{P_{r_{0}}}{u}}}\right], \\
\bar{v}_{2}(\psi, u)= & \frac{G r}{a}\left[-u-j+\frac{1}{h^{2}-g^{2}}\left\{D F_{4}(u)+E F_{5}(u)\right\}\right] F_{1}(u)\left[F_{2}(u)-F_{3}(u)\right], \\
\bar{v}_{2}(\psi, u)= & \frac{G r}{a}\left[-u F_{1}(u) F_{2}(u)+u F_{1}(u) F_{3}(u)-j\left(1-e^{-\frac{1}{u}}\right)\left(F_{2}(u)-F_{3}(u)\right)\right] \\
& +\frac{G r D}{a\left(h^{2}-g^{2}\right)}\left(1-e^{-\frac{1}{u}}\right) F_{4}(u)\left[F_{2}(u)-F_{3}(u)\right] \\
& +\frac{G r E}{a\left(h^{2}-g^{2}\right)}\left(1-e^{-\frac{1}{u}}\right) F_{5}(u)\left[F_{2}(u)-F_{3}(u)\right], \\
\bar{v}_{2}(\psi, u)= & \frac{G r}{a}\left[-S_{1}(u)+S_{2}(u)-j\left(F_{2}(u)-F_{3}(u)\right)-e^{-\frac{1}{u}} F_{2}(u)+e^{-\frac{1}{u}} F_{3}(u)\right] \\
& +\frac{G r D}{a\left(h^{2}-g^{2}\right)}\left[F_{24}(u)-F_{34}(u)-e^{-\frac{1}{u}} F_{24}(u)+e^{-\frac{1}{u}} F_{34}(u)\right] \\
& +\frac{G r E}{a\left(h^{2}-g^{2}\right)}\left[F_{25}(u)-F_{35}(u)-e^{-\frac{1}{u}} F_{25}(u)+e^{-\frac{1}{u}} F_{35}(u)\right],
\end{aligned}
$$

with

$$
\begin{aligned}
& j=\frac{P_{r_{0}}-c}{a}, \quad r=\frac{\alpha P_{r_{0}}}{a}, \quad z=j^{2}+r, \quad e_{1}=j . r, \quad g=\frac{j}{2}, \quad h=\sqrt{r+g^{2}}, \\
& d=c-a \alpha, \quad a_{2}=\frac{1}{h+g}, \quad b_{2}=\frac{1}{h-g}, \quad D=\frac{a_{2} e_{1}+z}{\left(a_{2}+b_{2}\right)}, \quad E=\frac{b_{2} e_{1}-z}{\left(a_{2}+b_{2}\right)}, \\
& c=1+b, \quad F_{1}(u)=1-e^{-\frac{1}{u}}, \quad F_{2}(u)=e^{-\psi \sqrt{\frac{a u+c}{u+\alpha}}}, \quad F_{3}(u)=e^{-\psi \sqrt{\frac{P r_{0}}{u}}}, \\
& F_{4}(u)=\frac{1}{1-a_{2} u}, \quad F_{5}(u)=\frac{1}{1+b_{2} u} .
\end{aligned}
$$

Employing the Sumudu inverse transformation, the solution is written as

$$
\begin{aligned}
v_{2}(\psi, t)= & \frac{G r}{a}\left[-S_{1}(\psi, t)+S_{2}(\psi, t)-j\left[f_{2}(\psi, t)-f_{3}(\psi, t)-f_{2}(\psi, t-1) H(t-1)\right.\right. \\
& \left.\left.+f_{3}(\psi, t-1) H(t-1)\right]\right]
\end{aligned}
$$




$$
\begin{aligned}
& +\frac{G r D}{a\left(h^{2}-g^{2}\right)}\left[f_{24}(\psi, t)-f_{34}(\psi, t)-f_{24}(\psi, t-1) H(t-1)\right. \\
& \left.+f_{34}(\psi, t-1) H(t-1)\right] \\
& +\frac{G r D}{a\left(h^{2}-g^{2}\right)}\left[f_{25}(\psi, t)-f_{35}(\psi, t)-f_{25}(\psi, t-1) H(t-1)\right. \\
& \left.+f_{35}(\psi, t-1) H(t-1)\right],
\end{aligned}
$$

where

$$
\begin{aligned}
& S_{1}(\psi, t)=\left(f_{1} * f_{2}\right)(t)=\int_{0}^{t} f_{1}(\tau) f_{2}(t-\tau) d(\tau), \\
& S_{2}(\psi, t)=\left(f_{1} * f_{3}\right)(t)=\int_{0}^{t} f_{1}(\tau) f_{3}(t-\tau) d(\tau), \\
& \mathbb{S}^{-1}\left(e^{-\frac{1}{u}} F_{2}(u)\right)=f_{2}(t-1) H(t-1), \\
& \mathbb{S}^{-1}\left(e^{-\frac{1}{u}} F_{3}(u)\right)=f_{3}(t-1) H(t-1),
\end{aligned}
$$

and

$$
\begin{aligned}
f_{3}(t)= & \operatorname{erfc}\left(\frac{a_{1}}{2 \sqrt{t}}\right), \quad \text { with } a_{1}=\sqrt{P_{r_{0}}} \psi, \\
F_{24}(u)= & F_{2}(u) F_{4}(u) \\
= & {\left[\sum_{n_{1}=0}^{\infty} \sum_{n_{2}=0}^{\infty} \sum_{n_{3}=0}^{\infty} \frac{(-1)^{n_{3}} a^{\frac{n_{1}}{2}-n_{2}}(-\psi)^{n_{1}} d^{n_{2}} \Gamma\left(\frac{n_{1}}{2}+1\right) \Gamma\left(n_{2}+n_{3}\right)}{n_{1} ! n_{2} ! n_{3} !(\alpha)^{n_{2}+n_{3}} \Gamma\left(\frac{n_{1}}{2}+1-n_{2}\right) \Gamma\left(n_{2}\right)} u^{n_{3}}\right] } \\
& \times\left[\sum_{l=0}^{\infty}\left(a_{2}(u)\right)^{l}\right] .
\end{aligned}
$$

Applying discrete convolution (the Cauchy product) with two truncated series, each of $m$ terms, yields:

$$
\begin{aligned}
&=\sum_{n_{1}=0}^{\infty} \sum_{n_{2}=0}^{\infty} \sum_{n_{3}=0}^{m} \sum_{l=0}^{m} \frac{(-1)^{n_{3}} a^{\frac{n_{1}}{2}-n_{2}}(-\psi)^{n_{1}} d^{n_{2}} \Gamma\left(\frac{n_{1}}{2}+1\right) \Gamma\left(n_{2}+n_{3}\right)}{n_{1} ! n_{2} ! n_{3} !(\alpha)^{n_{2}+n_{3}} \Gamma\left(\frac{n_{1}}{2}+1-n_{2}\right) \Gamma\left(n_{2}\right)} u^{n_{3}}\left(a_{2}(u)\right)^{m-l}, \\
& f_{24}(\psi, t)= \mathbb{S}^{-1}\left(F_{24}(u)\right) \\
&= \sum_{n_{1}=0}^{\infty} \sum_{n_{2}=0}^{\infty} \sum_{n_{3}=0}^{m} \sum_{l=0}^{m} \frac{(-1)^{n_{3}} a^{\frac{n_{1}}{2}-n_{2}}(-\psi)^{n_{1}} d^{n_{2}} \Gamma\left(\frac{n_{1}}{2}+1\right) \Gamma\left(n_{2}+n_{3}\right)}{n_{1} ! n_{2} !\left(n_{3} !\right)^{2}(\alpha)^{n_{2}+n_{3}} \Gamma\left(\frac{n_{1}}{2}+1-n_{2}\right) \Gamma\left(n_{2}\right)} \\
& \cdot \frac{t^{n_{3}+m-l}}{\left(n_{3}+m-l\right) !}\left(a_{2}\right)^{m-l}, \\
& F_{34}(u)= F_{3}(u) F_{4}(u) \\
&= {\left[\sum_{\beta=0}^{\infty}\left(\frac{-a_{1}}{\sqrt{u}}\right)^{\beta} \frac{1}{\beta !}\right]\left[\sum_{\eta=0}^{\infty}\left(a_{2}(u)\right)^{\eta}\right] . }
\end{aligned}
$$


By using Cauchy product or discrete convolution, we get the product of the above two series as a truncated double series

$$
\begin{aligned}
& =\sum_{\beta=0}^{\mu} \sum_{\eta=0}^{\mu} \frac{\left(-a_{1}\right)^{\beta}\left(a_{2}\right)^{\mu-\eta}}{\beta !}(u)^{\frac{-\beta}{2}}(u)^{\mu-\eta}, \\
& =\sum_{\beta=0}^{\mu} \sum_{\eta=0}^{\mu} \frac{\left(-a_{1}\right)^{\beta}\left(a_{2}\right)^{\mu-\eta}}{\beta !}(u)^{\mu-\eta-\frac{\beta}{2}}, \\
& f_{34}(\psi, t)=\mathbb{S}^{-1}\left(F_{34}(u)\right), \\
& =\sum_{\beta=0}^{\mu} \sum_{\eta=0}^{\mu} \frac{\left(-a_{1}\right)^{\beta}\left(a_{2}\right)^{\mu-\eta}}{\beta ! \Gamma\left(\mu-\eta-\frac{\beta}{2}+1\right)}(t)^{\mu-\eta-\frac{\beta}{2}}, \\
& \mathbb{S}^{-1}\left(e^{-\frac{1}{u}} F_{24}(u)\right)=f_{24}(t-1) H(t-1), \\
& \mathbb{S}^{-1}\left(e^{-\frac{1}{u}} F_{34}(u)\right)=f_{34}(t-1) H(t-1), \\
& f_{25}(\psi, t)=\sum_{n_{1}=0}^{\infty} \sum_{n_{2}=0}^{\infty} \sum_{n_{3}=0}^{i} \sum_{w=0}^{i} \frac{(-1)^{n_{3}} a^{\frac{n_{1}}{2}-n_{2}}(-\psi)^{n_{1}} d^{n_{2}} \Gamma\left(\frac{n_{1}}{2}+1\right) \Gamma\left(n_{2}+n_{3}\right)}{n_{1} ! n_{2} !\left(n_{3} !\right)^{2}(\alpha)^{n_{2}+n_{3}} \Gamma\left(\frac{n_{1}}{2}+1-n_{2}\right) \Gamma\left(n_{2}\right)} \\
& \quad \times \frac{t^{n_{3}+i-w}}{\left(n_{3}+i-w\right) !}\left(-b_{2}\right)^{i-w}, \\
& f_{35}(\psi, t)=\sum_{\beta_{1}=0}^{\mu_{1}} \sum_{\eta_{1}=0}^{\mu_{1}} \frac{\left(-a_{1}\right)^{\beta_{1}}\left(-b_{2}\right)^{\mu_{1}-\eta_{1}}}{\left(\beta_{1}\right) ! \Gamma\left(\mu_{1}-\eta_{1}-\frac{\beta_{1}}{2}+1\right)}(t)^{\mu_{1}-\eta_{1}-\frac{\beta_{1}}{2}}, \\
& \mathbb{S}^{-1}\left(e^{-\frac{1}{u}} F_{25}(u)\right)=f_{25}(t-1) H(t-1), \\
& \mathbb{S}^{-1}\left(e^{-\frac{1}{u}} F_{35}(u)\right)=f_{35}(t-1) H(t-1) .
\end{aligned}
$$

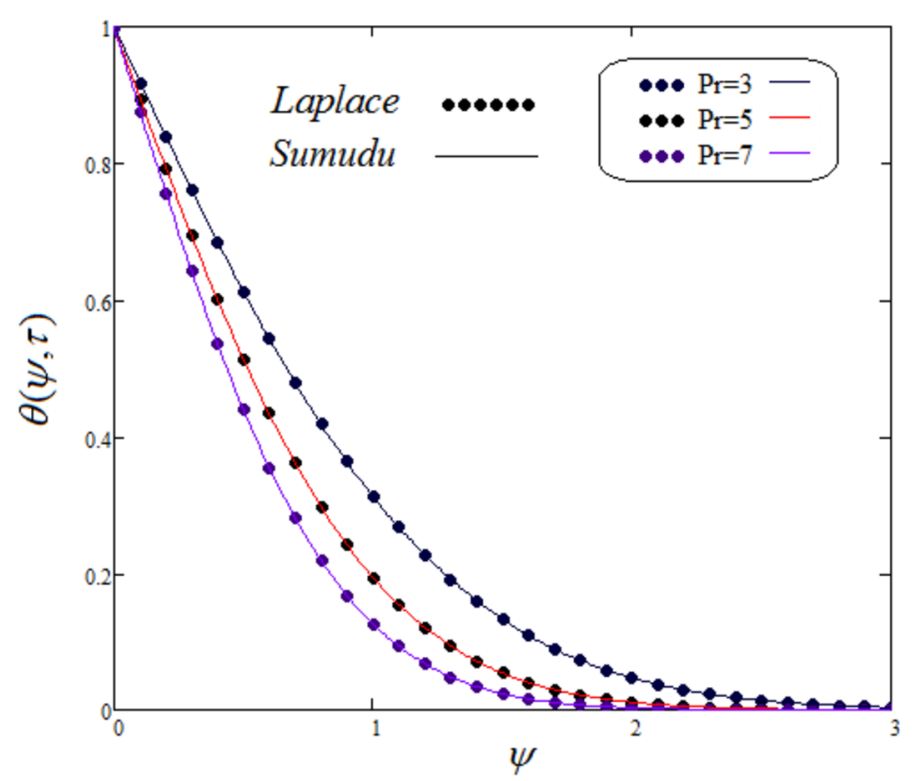

Figure 1 Temperature profile for different values of Pr via Laplace and Sumudu transformation 


\section{Results and discussion}

From Eqs. (34) and (41), we observe that the temperature profile has two different solution expressions calculated by Sumudu transformation in (34) and by Laplace transformation method in (41). These are graphically equivalent. Figure 1 presents the temperature illustration for various values of $P r$. It has been declared that when the values of $P r$ increase, the temperature is falling in both cases.

\section{Conclusion}

We presented a new application of the Sumudu transform in this paper. The Sumudu transform is able to keep the unity of the function, the parity of the function, and has many other properties that are more valuable. Therefore, we investigated the Sumudu transform in this work. We compared the results with the results obtained by the Laplace transform. We proved the efficiency of the Sumudu transform for solutions of the unsteady convective flow of an MHD second grade fluid with ramped conditions.

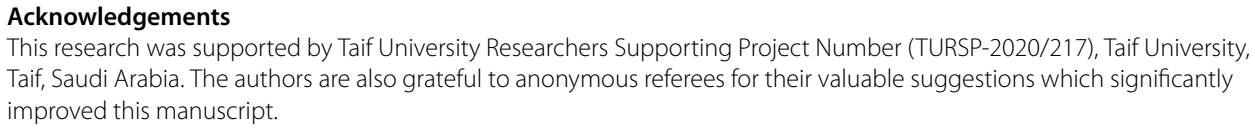

This research was supported by Taif University Researchers Supporting Project Number (TURSP-2020/217), Taif University, Taif, Saudi Arabia. The authors are also grateful to anonymous referees for their valuable suggestions which significantly improved this manuscript.

\section{Funding}

No external funding was available for this research.

Availability of data and materials

Not applicable.

\section{Competing interests}

The authors declare that they have no competing interests.

\section{Authors' contributions}

All authors equally contributed to this work. All authors read and approved the final manuscript.

\section{Author details}

${ }^{1}$ Department of Mathematics, University of Management and Technology, Lahore, Pakistan. ${ }^{2}$ Institute for Groundwater Studies (IGS), University of the Free State, Bloemfontein, South Africa. ${ }^{3}$ Department of Basic Sciences and Related Studies, Mehran University of Engineering and Technology, Jamshoro, Pakistan. ${ }^{4}$ Department of Mathematics and Statistics, College of Science, Taif University, Taif 21944, Saudi Arabia. ${ }^{5}$ Department of Mathematics, Art and Science Faculty, Siirt University, TR-56100 Siirt, Turkey. ${ }^{6}$ Department of Mathematics, University of Sargodha, 40100 Sargodha, Pakistan.

\section{Publisher's Note}

Springer Nature remains neutral with regard to jurisdictional claims in published maps and institutional affiliations.

Received: 21 June 2021 Accepted: 24 August 2021 Published online: 08 September 2021

\section{References}

1. Ganesan, P., Palani, G.: Natural convection effects on impulsively started inclined plate with heat and mass transfer. Heat Mass Transf. 39, 277-283 (2003)

2. UI Haq, S., Fetecau, C., Khan, I., Ali, F., Shafie, S.: Radiation and porosity effects on the magnetohydrodynamic flow past an oscillating vertical plate with uniform heat flux. Z. Naturforsch. 67a, 572-580 (2012)

3. Chandran, P., Sacheti, N.C., Singh, A.K.: Natural convection near a vertical plate with ramped wall temperature. Heat Mass Transf. 41, 459-464 (2005)

4. Seth, G.S., Ansari, M.D.S.: MHD natural convection flow past an impulsively moving vertical plate with ramped wall temperature in the presence of thermal diffusion with heat absorption. Appl. Mech. Eng. 15, 199-215 (2010)

5. Seth, G.S., Ansari, M.D.S., Nandkeolyar, R.: MHD natural convection flow with radiative heat transfer past an impulsively moving plate with ramped wall temperature. Heat Mass Transf. 47, 551-561 (2011)

6. Atangana, A., Akgül, A.: Can transfer function and Bode diagram be obtained from Sumudu transform. Alex. Eng. J. 59(4), 1971-1984 (2020)

7. Watugala, G.K.: Sumudu transform: a new integral transform to solve differential equations and control engineering problems. Integr. Educ. 24(1), 35-43 (1993)

8. Weerakoon, S.: Application of Sumudu transform to partial differential equations. Int. J. Math. Educ. Sci. Technol. 25(2), 277-283 (1994) 
9. Weerakoon, S.: Complex inversion formula for Sumudu transform. Int. J. Math. Educ. Sci. Technol. 29(4), 618-620 (1998)

10. Asiru, M.A.: Sumudu transform and the solution of integral equations of convolution type. Int. J. Math. Educ. Sci. Technol. 32(6), 906-910 (2001)

11. Watugala, G.K.: The Sumudu transform for functions of two variables. Math. Eng. Ind. 8(4), 293-302 (2002)

12. Belgacem, F.B., Karaballi, A.A., Kalla, S.L.: Analytical investigations of the Sumudu transform and applications to integral production equations. Math. Probl. Eng. 2003, Article ID 439059 (2003)

13. Belgacem, F.B., Karaballi, A.A.: Sumudu transform fundamental properties investigations and applications. Int. J. Stoch. Anal. 2006, Article ID 091083 (2006)

14. Demiray, S.T., Bulut, H., Belgacem, F.B.: Sumudu transform method for analytical solutions of fractional type ordinary differential equations. Math. Probl. Eng. 2015, Article ID 131690 (2015)

15. Maritz, R., Goufo, E.F.D.: Newtonian and non-Newtonian fluids through permeable boundaries. Math. Probl. Eng. 2014, Article ID 146521 (2014). https://doi.org/10.1155/2014/146521

16. Doungmo Goufo, E.F., Kumar, S., Mugisha, S.B.: Similarities in a fifth-order evolution equation with and with no singular kernel. Chaos Solitons Fractals 130, 109467 (2020). https://doi.org/10.1016/j.chaos.2019.109467

17. Goufo, E.F.D.: The Proto-Lorenz system in its chaotic fractional and fractal structure. Int. J. Bifurc. Chaos 30, 2050180 (2020). https://doi.org/10.1142/S0218127420501801

18. Goufo, E.F.D.: Fractal and fractional dynamics for a 3D autonomous and two-wing smooth chaotic system. Alex. Eng. J. 59, 2469-2476 (2020). https://doi.org/10.1016/.aej.2020.03.011

19. Bardos, C., Golse, F., Pertham, B.: The Rosseland approximation for the radiative transfer equations. Commun. Pure Appl. Math. 40, 691-721 (1987)

20. Khan, I., Ellahi, R., Fetecau, C.: Some MHD flows of a second grade fluid through the porous medium. J. Porous Media $11,389-400(2008)$

21. Rehman, A.U., Riaz, M.B., Awrejcewicz, J., Baleanu, D.: Exact solutions of thermomagetized unsteady non-singularized Jeffrey fluid: effects of ramped velocity,concentration with Newtonian heating. Results Phys. 26, 104367 (2021)

22. Imran, M.A., Riaz, M.B., Shah, N.A., Zafar, A.A.: Boundary layer flow of MHD generalized Maxwell fluid over an exponentially accelerated infinite vertical surface with slip and Newtonian heating at the boundary. Results Phys. 8 , 1061-1067 (2018)

\section{Submit your manuscript to a SpringerOpen ${ }^{\circ}$ journal and benefit from:}

- Convenient online submission

- Rigorous peer review

- Open access: articles freely available online

- High visibility within the field

Retaining the copyright to your article

Submit your next manuscript at $\boldsymbol{~ s p r i n g e r o p e n . c o m ~}$ 